Research Article

\title{
Fault Detection for Turbine Engine Disk Based on Adaptive Weighted One-Class Support Vector Machine
}

\author{
Jiusheng Chen $(D$, Xingkai Xu, and Xiaoyu Zhang $(i D)$ \\ College of Electronic Information and Automation, Civil Aviation University of China, Tianjin 300300, China \\ Correspondence should be addressed to Jiusheng Chen; jschen@cauc.edu.cn
}

Received 21 May 2020; Revised 26 July 2020; Accepted 7 August 2020; Published 28 August 2020

Academic Editor: James Lam

Copyright (c) 2020 Jiusheng Chen et al. This is an open access article distributed under the Creative Commons Attribution License, which permits unrestricted use, distribution, and reproduction in any medium, provided the original work is properly cited.

\begin{abstract}
Fault detection for turbine engine components is becoming increasingly important for the efficient running of commercial aircraft. Recently, the support vector machine (SVM) with kernel function is the most popular technique for monitoring nonlinear processes, which can better handle the nonlinear representation of fault detection of turbine engine disk. In this paper, an adaptive weighted one-class SVM-based fault detection method coupled with incremental and decremental strategy is proposed, which can efficiently solve the time series data stream drifting problem. To update the efficient training of the fault detection model, the incremental strategy based on the new incoming data and support vectors is proposed. The weight of the training sample is updated by the variations of the decision boundaries. Meanwhile, to increase the calculating speed of the fault detection model and reduce the redundant data, the decremental strategy based on the $k$-nearest neighbor (KNN) is adopted. Based on time series data stream, numerical simulations are conducted and the results validated the superiority of the proposed approach in terms of both the detection performance and robustness.
\end{abstract}

\section{Introduction}

The turbine engine is the core component of the aircraft's propulsion system. During the flight, if the engine fails, it will find a serious threat to safe flight. Due to the complex working environment of the engine, the working status of the engine rotor system determines the reliability and safety of an aircraft engine $[1,2]$. In the daily operation of commercial aircraft, minor cracks and hidden flaws in the turbine engine disk can lead to catastrophic events. In particular, when early failures occur, timely capture of failure information in order to take effective diagnosis and maintenance measures is of great significance for preventing and eliminating hidden dangers of malignant accidents and reducing or even avoiding general failures [3]. Predictive maintenance based on condition monitoring is to periodically or continuously monitor the condition of the equipment and, according to the results of condition monitoring and fault diagnosis, find out whether the equipment has deterioration or failure signs and then arrange maintenance. It can more accurately estimate the reliable running time, reduce the consumption of spare parts and maintenance workload, also prevent man-made failures due to maintenance, and ultimately reduce maintenance costs. After the implementation of condition monitoring and maintenance, the maintenance time is reduced, and the production efficiency and economic benefits are improved [4].

In order to tackle the problem, fault detection technique could prevent the occurrence of any serious affair. Driven by the increasing demand for reliability and safety, the fault detection technique in the turbine engine disk is a critical scientific issue. And how to design a fault detection system with high accuracy has become a widely concerned topic $[5,6]$. The data-driven method can analyze the process data and extract necessary information from huge amounts of historical offline data to complete the fault detection of the turbine engine disk without modeling. Throughout the development of data-driven fault detection, diagnosis, and recognition technology, it has been widely studied and applied in academia and industry.

Data-driven approaches mainly rely on analyzing process data and extracting features from them without 
modeling a sophisticated system, so the data-driven fault detection technology can be easily applied. The current mainstream data-driven fault detection algorithms are mainly based on machine learning methods, which mainly include artificial neural network (ANN) [7, 8], Gaussian Process (GP) [9, 10], Random Forest [11], and support vector machines (SVMs) [12].

An ANN-based approach was put forward for incipient gearbox fault diagnosis, in which the fault features were extracted by discrete wavelet transform (DWT), and the faults in a gearbox were detected by the ANN model [7]. The experimental results showed that the approach could reliably detect the faults of the gearbox. A GP-based condition monitoring approach was presented to predict the blade pitch curve of a wind turbine $[9,10]$. GP model is a robust method for approximating complex nonlinear models, which can estimate the magnitude of the relevant uncertainty and calculate the confidence interval, respectively. Experimental results showed that the GP-based condition monitoring approach could be useful for monitoring the power performance assessment of wind turbines. A deep random forest fusion (DRFF) technique was adopted in the fault diagnosis of gearboxes by Li et al. [11]. By adopting a deep learning feature representation and data fusion strategy, the performance of fault diagnosis was improved.

SVM is a powerful data-driven machine learning method, which is widely used in classification and regression related problems. Different from the existing statistical methods, the SVM algorithm adopts the principle of structural risk minimization, and it has particularly good generalization performance [12]. Therefore, the SVM-based approach has been widely used in the field of fault detection and condition monitoring. A combination of SVM and ANN was adopted in the fault detection of rotating machinery by Jack et al. [13], which can improve the detection performance with limited training data. An SVM-based fault detection model with radial basis function (RBF) kernel function was presented to fault detection of the wind turbine $[14,15]$. The experimental results show that the approach can efficiently monitor the operation condition of the wind turbine.

Most of traditional SVM-based fault detection models focus on supervised learning, which means that fully labeled datasets are needed to build the detection model. However, plenty of normal samples of turbine engine disks can be acquired easily, while the fault samples of turbine engine disks are difficult to obtain or even not available, which limits the real-life applications of supervised learning methods. Meantime, the operation of the engine turbine disk is a time-varying and nonstationary process which has a nonlinear dynamical behavior. In order to solve the above problem, an adaptive one-class support vector machine (SVM) is particularly proposed and widely used in the fault detection system $[16,17]$. In order to analyze the data stream with concept drift, an incremental weighted one-class SVM was proposed by Krawczyk and Woźniak, in which the incremental learning is used to distribute the weight of the new sample to change the shape of the previously learned decision boundary and the incremental forgetting strategy is used to accelerate the computing performance $[18,19]$.

In this paper, we proposed a novel algorithm for learning an adaptive weighted one-class SVM for fault detection of the turbine engine disk. The major advantages lie in two aspects. Firstly, an incremental learning mechanism is proposed for the time series fault detection of the turbine engine disk, which can efficiently deal with the time series data stream with the drifting problem. Secondly, a decremental strategy based on $k$-nearest neighbor (KNN) is used to increase the calculating speed of the fault detection model while remaining the correct results. A pair of samples with a maximum Euclidean distance of the nearest neighbor is preserved by the decremental strategy based on KNN . Consequently, the basic structure of input samples is preserved, which can improve the robustness of the algorithm.

The article is organized as follows. The adaptive fault detection technique for a nonstationary process is introduced in Section 1. A review of the weighted one-class SVM is listed in Section 2. The proposed adaptive weighted oneclass SVM-based fault detection with incremental and decremental strategy is discussed in Section 3. The simulation is conducted and the performance of the proposed method is thoroughly examined in Section 4 . The conclusion is given in Section 5.

\section{Weighted One-Class SVM}

The weighted one-class SVM [20] is proposed by Bicego and Figueiredo which is the extension of a one-class support vector machine (OCSVM) proposed by Schölkopf et al. [21] and one-class SVDD proposed by Tax and Duin [22]. The weighted OCSVM and one-class SVDD are briefly introduced as follows.

2.1. Weighted OCSVM. OCSVM was proposed by Schölkopf et al. to solve the novelty detection problem, which is an unsupervised learning method for constructing a separating hyperplane that can separate the normal and abnormal samples in the feature space. The weighted OCSVM introduces a vector of weight variables $\omega=\left[\omega_{1}, \omega_{2}, \ldots, \omega_{\ell}\right]^{T}$, where $\omega_{i} \in[0,1], \quad i=1,2, \cdots$, and $\ell$ is the importance assigned to each training sample. Referring to the optimization model of OCSVM, the weighted OCSVM can be formulated as follows:

$$
\begin{array}{ll}
\min _{w, \rho, \xi} & \frac{1}{2}\|w\|^{2}-\rho+\frac{1}{v \ell} \sum_{i=1}^{\ell} \omega_{i} \xi_{i} \\
\text { s.t. } & \mathbf{w}^{T} \phi\left(x_{i}\right) \geq \rho-\xi_{i}, \quad i=1, \ldots, \ell \\
& \xi_{i} \geq 0, \quad i=1, \ldots, \ell,
\end{array}
$$

where $\mathbf{w}$ is the normal vector of the separating hyperplane, $\rho$ is the margin, $\xi=\left[\xi_{1}, \ldots, \xi_{l}\right]^{T}$ is the vector of slack variables, and $\nu$ is a positive constant. The dual form of weighted OCSVM can be obtained as follows: 


$$
\begin{array}{ll}
\min _{\boldsymbol{\alpha}} & \frac{1}{2} \boldsymbol{a}^{\mathrm{T}} \mathbf{K} \boldsymbol{\alpha} \\
\text { s.t. } & 0 \leq \alpha_{i} \leq \frac{\omega_{i}}{(\nu \ell)}, \quad i=1, \ldots, \ell \\
& \sum_{i=1}^{\ell} \alpha_{i}=1, \quad i=1, \ldots, \ell,
\end{array}
$$

where $\mathbf{K}$ is the kernel matrix and $\alpha=\left[\alpha_{1}, \ldots, \alpha_{\ell}\right]^{T}$ is the vector of Lagrange multiplier. To classify a testing instance $\mathbf{z} \in R^{d}$, the decision function of weighted OCSVM can be calculated as follows:

$$
f(\mathbf{z})=\operatorname{sgn}\left(\sum_{i=1}^{\ell} \alpha_{i} K\left(\mathbf{x}_{i}, \mathbf{z}\right)-\rho\right),
$$

where $K(\cdot, \cdot)$ is the kernel function.

2.2. Weighted One-Class SVDD. One-class SVDD is proposed by Tax et al., in which a minimal ball is constructed in the form of a hypersphere enclosing most of the training samples. The normal samples fall within the hypersphere of the feature space. Otherwise, the outlier samples fall outside the hypersphere of the feature space. The weighted one-class SVDD introduces a vector of weight variables $\omega=\left[\omega_{1}, \omega_{2}, \ldots, \omega_{\ell}\right]^{T}$, where $\omega_{i} \in[0,1], \quad i=1,2, \cdots$, and $\ell$ is the importance assigned to each training sample. Referring to the optimization model of one-class SVDD, the weighted one-class SVDD can be formulated as follows:

$$
\begin{array}{ll}
\min _{R, \mathbf{c}, \xi} & R^{2}+C \sum_{i=1}^{\ell} \omega_{i} \xi_{i} \\
\text { s.t. } & \left\|\phi\left(\mathbf{x}_{i}\right)-\mathbf{c}\right\|^{2} \leq R^{2}+\xi_{i}, \quad i=1, \ldots, \ell \\
& \xi_{i} \geq 0, \quad i=1, \ldots, \ell,
\end{array}
$$

where $\mathbf{c}$ and $R$ are the center and radius of the separating hypersphere, respectively, $\xi=\left[\xi_{1}, \ldots, \xi_{\ell}\right]^{T}$ is the vector of slack variables, $C$ is the regularization parameter, and $\|\cdot\|$ is the Euclidean norm. The dual form of weighted one-class SVDD can be obtained as follows:

$$
\begin{array}{ll}
\min _{\alpha} & \boldsymbol{\alpha}^{T} \mathbf{K} \boldsymbol{\alpha}-\boldsymbol{\alpha}^{T} \operatorname{diag}(\mathbf{K}) \\
\text { s.t. } & 0 \leq \alpha_{i} \leq \omega_{i} C, \quad i=1, \ldots, \ell \\
& \sum_{i=1}^{\ell} \alpha_{i}=1, \quad i=1, \ldots, \ell,
\end{array}
$$

where $\mathbf{K}$ is the kernel matrix, $\operatorname{diag}(\mathbf{K})$ is the diagonal elements of the kernel matrix, and $\alpha=\left[\alpha_{1}, \ldots, \alpha_{\ell}\right]^{T}$ is the vector of Lagrange multipliers. In order to classify a testing instance $\mathbf{z} \in R^{d}$, the decision function of weighted one-class SVDD can be calculated as follows:

$$
f(\mathbf{z})=\operatorname{sgn}\left(R^{2}-\left\|\phi\left(\mathbf{x}_{i}\right)-\mathbf{c}\right\|^{2}\right) .
$$

\section{Fault Detection of Turbine Engine Disk Based on Adaptive Weighted One-Class SVM}

The working process of the turbine engine disk is timevarying. Each time series of turbine engine disks could not be analyzed separately in the fault detection model. In order to improve the fault detection capability effect of the time series sample in a long sequence, an adaptive weighted one-class SVM-based fault detection method with incremental and decremental strategy is interpreted in Figure 1.

In the adaptive weighted one-class SVM-based fault detection method, an incremental learning strategy is introduced to update the weighted one-class SVM fault detection depending on the support vectors and the latest samples. However, with the work of the fault detection model, increasing incoming samples will be added under the scheme of the incremental learning strategy. If the training samples increase just by incremental learning strategy, it will lead to the dimension disaster. Therefore, a decremental learning strategy is introduced to reduce redundant data.

3.1. The Incremental Strategy of Weighted One-Class SVM. Suppose that $\mathrm{TDS}_{t}$ is the training dataset at $t-$ th iteration, $\mathrm{WDS}_{t}$ is the support vectors at $t$ - th iteration, $\mathrm{IDS}_{t}$ is the new increase samples at $t$-th iteration, and NIDS $_{t}$ is the normal samples of $\operatorname{IDS}_{t}$ at $t$ - th iteration.

The incremental strategy of weighted one-class SVM can be expressed as follows:

$$
\mathrm{TDS}_{t}=\mathrm{WDS}_{t-1} \cup \mathrm{NIDS}_{t}
$$

where $\mathrm{WDS}_{t-1}$ is the support vectors dataset at $(t-1)-$ th iteration which represents historical decision information and NIDS $_{t}$ is the new increased dataset which represents the current state of the turbine engine disk.

In this work, the radial basis function $(\mathrm{RBF})$ is taken as the kernel function:

$$
K\left(\mathbf{x}_{i}, \mathbf{x}_{j}\right)=\exp \left(-\gamma\left\|\mathbf{x}_{i}-\mathbf{x}_{j}\right\|^{2}\right), \quad 0<\gamma<+\infty
$$

where $\gamma$ is the kernel parameter of the RBF. From the RBF kernel function, it is found that if the discrimination between $\mathbf{x}_{i}$ and $\mathbf{x}_{j}$ is greater, (1) $K\left(\mathbf{x}_{i}, \mathbf{x}_{j}\right) \approx 1$, i.e., the distance of two training samples $\mathbf{x}_{i}$ and $\mathbf{x}_{j}$ in the feature space approaches 0 , and $(2) K\left(\mathbf{x}_{i}, \mathbf{x}_{j}\right) \approx 0$, i.e., the distance of two training samples $\mathbf{x}_{i}$ and $\mathbf{x}_{j}$ in the feature space approaches $+\infty$. Therefore, the similarity between samples can be measured by the RBF kernel function.

For the SVM-based fault detection model, the decision function is determined by support vectors. In this paper, we introduce a novel method to calculate the sample weight as follows:

$$
\omega_{i}=1-K\left(x_{i}, x_{\text {mean }}\right)=1-\exp \left(-\gamma\left\|x_{i}-x_{\text {mean }}\right\|^{2}\right),
$$

where $\mathbf{x}_{\text {mean }}$ is the mean of the support vectors dataset $\mathrm{WDS}_{t-1}$. From (9), we can find that the distance between the sample and decision boundary increases with the weight of the sample. Significant weight samples represent the change 


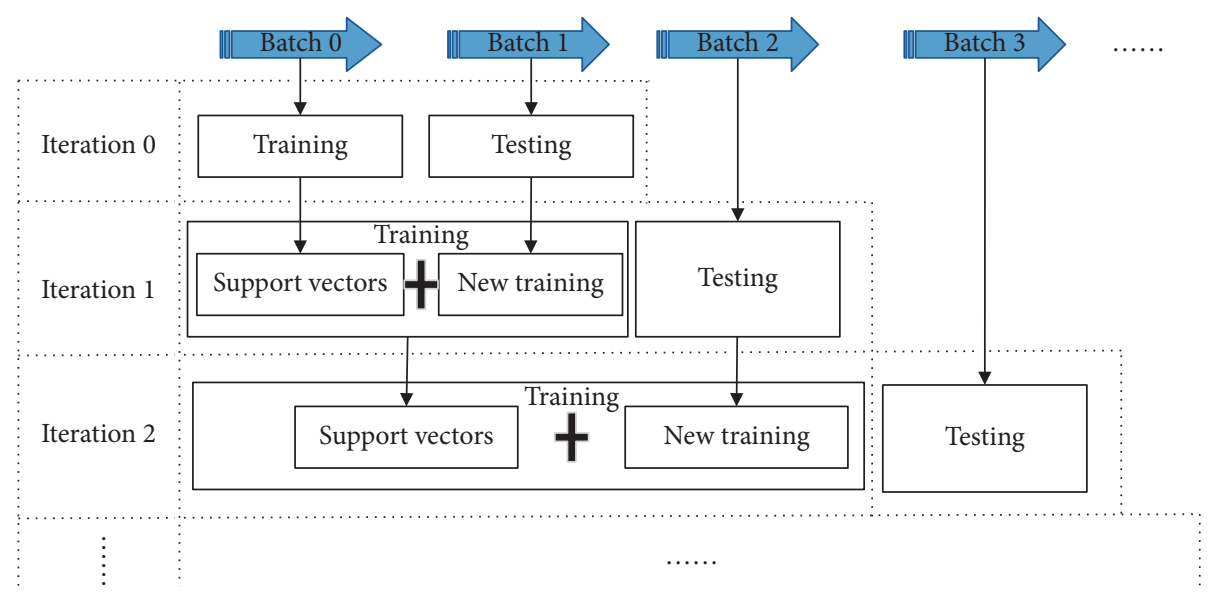

FigURE 1: Working process of adaptive weighted one-class SVM.

in decision boundary and, consequently, a significant impact on the new decision boundary.

The flow diagram incremental strategy of weighted oneclass SVM is shown in Figure 2.

(1) Initialize the training dataset $\mathrm{TDS}_{0}$ and set $t=0$

(2) Calculate the weight of the sample according to equation (9), and solve the weighted one-class SVM problem equation (2) or equation (5) by the training dataset $\mathrm{TDS}_{t}$

(3) Obtain the weighted one-class SVM-based fault detection model, and store the support vectors dataset as WDS,

(4) Collect new testing data chunk, and classify the test pattern by the decision function equation (3) or equation (6)

(5) If there exist fault samples, a fault alarm should be given; otherwise, go to step 7

(6) If the process of novelty detection is ending, go to step 10; otherwise, go to step 7

(7) Collect new training dataset IDS $_{t}$ and update $t=t+1$

(8) Update the training dataset $\mathrm{TDS}_{t}$, and remove the redundant samples of $\mathrm{TDS}_{t}$ by the decremental strategy of weighted one-class SVM

(9) Go back to step 2

(10) End of fault detection

3.2. The Decremental Strategy of Weighted One-Class SVM. If only the incremental strategy is applied to the weighted one-class SVM, the dimension of the kernel matrix will increase which is impractical. We propose a redundant data deletion algorithm based on KNN for the KPCA method [23]. In the decremental strategy based on the KNN method, the samples with the maximum Euclidean distance are preserved by the KNN framework and the samples with smaller Euclidean distance are discarded. Therefore, the basic structure of input samples can be preserved and the variance of the reduced dataset becomes larger, which will improve the robustness of the algorithm on a small dataset. The flow diagram of the decremental strategy based on KNN is given in Figure 3:

The decremental strategy of weighted one-class SVM is accomplished by conducting the following steps sequentially:

(1) Initialize the input training dataset $\mathrm{TDS}_{t}, n$ is the number of $\mathrm{TDS}_{t}$, and set $i=0$

(2) Calculate the $k$-nearest neighbor of the sample $\mathbf{x}_{i}\left(\mathbf{x}_{i} \in \mathrm{TDS}_{t}\right)$

(3) Find the samples $\mathbf{x}_{m}$ and $\mathbf{x}_{n}$ with the maximum Euclidean distance in the $k$-nearest neighbor of the sample $\mathbf{x}_{i}\left(\mathbf{x}_{i} \in \mathrm{TDS}_{t}\right)$

(4) Store the samples $\mathbf{x}_{m}$ and $\mathbf{x}_{n}$ into the subset of the input training dataset $\operatorname{STDS}_{t}$ without reduplicate sample

(5) $i=i+1$. If $i>n$, go to step 6 ; otherwise, go to step 2

(6) Output the subset of the input training dataset STDS $_{t}$

\section{Experimental Results}

The performance of the proposed adaptive fault detection algorithm is evaluated on Disk Defect Data [5] which was collected by the National Aeronautics and Space Administration (NASA) Glenn Research Center's Rotor Dynamics Laboratory. Three typical states, normal, notch, and large notch, are recorded, respectively, in Disk Defect Data.

All the experiments were conducted on a computer with a Xeon (R) W-2133 at $3.60 \mathrm{GHz} \mathrm{CPU}$ and a $32-\mathrm{GB}$ main memory. Accuracy rate (AR), false alarm rate (FAR), training time, and testing time are used to evaluate the performance of the proposed fault detection method. All the experiments are implemented by the LIBSVM package [24, 25].

4.1. Parameters Selection. In all simulations, regularization parameters $\gamma, C$, and $\nu$ are selected by 5 -fold cross-validation. The kernel parameter $\gamma$ of the RBF is selected in

$$
\left\{\frac{\alpha_{0}}{128}, \frac{\alpha_{0}}{64}, \frac{\alpha_{0}}{32}, \frac{\alpha_{0}}{16}, \frac{\alpha_{0}}{8}, \frac{\alpha_{0}}{4}, \frac{\alpha_{0}}{2}, \alpha_{0}\right\},
$$




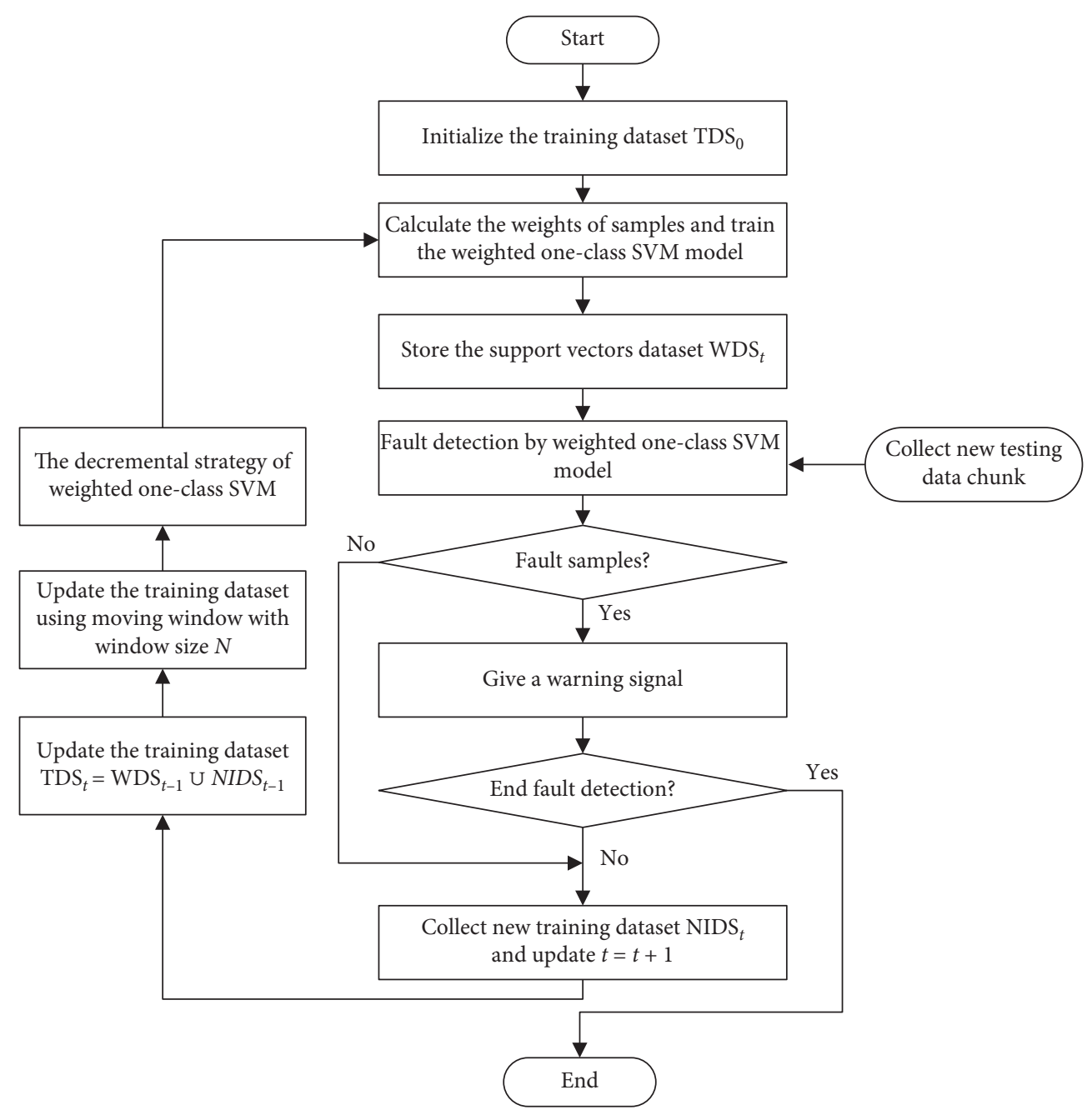

FIgURE 2: Flow diagram of the incremental strategy.

where $\alpha_{0}$ is the average norm of the training samples. The regularization parameter $C$ of weighted one-class SVDD is searched in $\{0.01 k, 0.1 k\}, k=1,3,5,7,9$. Similarly, the regularization parameter $v$ of weighted OCSVM is searched in $\{0.01 k, 0.1 k\}, k=1,3,5,7,9$.

The number of nearest neighbors is set to 6 . The number of initial training samples is 150 . The size of the new incoming chunk is 200. The size of the fixed moving window is 400 .

\subsection{Detection Performance of Adaptive Weighted OCSVM.} In this case, the turbine engine disk operates in normal working mode for 9000 samples with a rotating speed of 5000 RPM. After normal working mode, the notch model occurs on the turbine engine disk for 9000 samples (3000 samples with a rotating speed of 5000 RPM, 4000 RPM, and 3000 RPM, respectively).

Several kinds of fault detection methods, that is, static weighted OCSVM, adaptive weighted OCSVM with fixed moving window, and adaptive weighted OCSVM with incremental and decremental strategy, are compared. For the static weighted OCSVM, the fault detection model is trained without any adaptation mechanism. The weight of the normal sample coming from the new incoming chunk is assigned to 1 .

For adaptive weighted OCSVM with a fixed moving window, the fault detection model is trained by an adaptation mechanism through a moving window with a fixed length of 400 . The weight of the normal sample coming from the new incoming chunk is calculated by equation (9).

For the adaptive weighted OCSVM with incremental and decremental strategy, the fault detection model is trained by an adaptation mechanism in which the incremental strategy is assigned in Section 3.1 and a decremental strategy is assigned in Section 3.2.

The average and standard deviation of AR and FAR, the average training time, and the average testing time of the comparative algorithms are recorded in Table 1, in which the result shown in boldface is not significantly different from the BEST one, which is determined by the Wilcoxon ranksum test, while the confidence level is 0.05 . Additionally, the detailed AR and FAR behaviors of the different fault detection models on each data chunk are shown in Figure 4.

From Table 1 and Figure 4, it is observed that the detection performance of adaptive weighted OCSVM with incremental and decremental strategy is compared favorably 


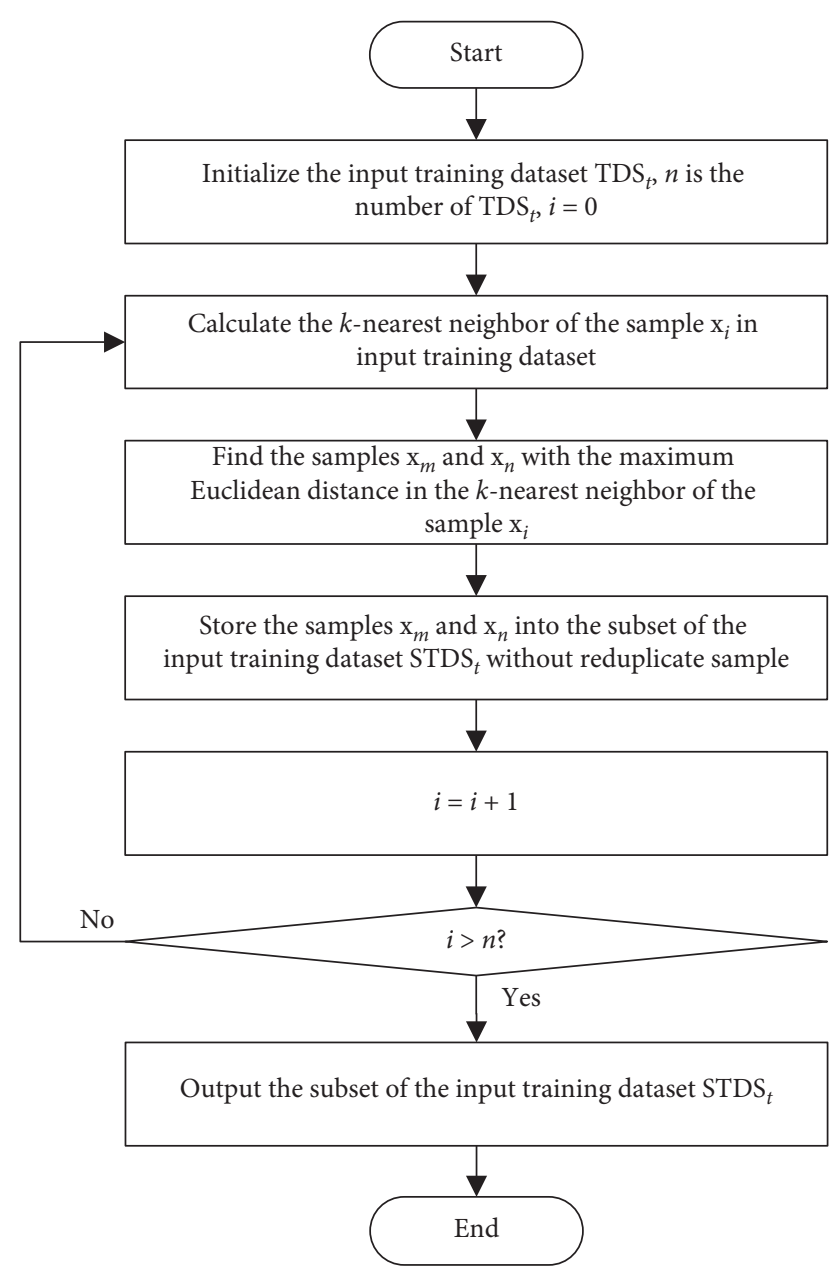

FIgURE 3: Flow diagram of the decremental strategy.

to the other algorithms. In general, it achieves the best performance of all methods.

From Figure 4(a), we can see that the detection accuracy rate is almost $100 \%$ after 9000 testing samples. After 9000 testing samples, there is a notch in the turbine engine disk, which operates in abnormal working mode. The OCSVMbased fault detection model is an unsupervised learning method for constructing a separating hyperplane which means that only normal samples are used to build the detection model. Therefore, by training enough normal samples for OCSVM, the detection rate of abnormal samples can be effectively improved.

From Table 1, it can be seen that the average AR of three weighted OCSVM-based fault detection methods is $91.0 \%$, $98.0 \%$, and $99.1 \%$, respectively. The average AR of the proposed method is about $8.1 \%$ and $1.1 \%$ higher than that of the static model and adaptive model with a fixed moving window, respectively. The FAR of three weighted OCSVMbased fault detection methods is $18.0 \%, 4.2 \%$, and $1.8 \%$, respectively. The average FAR of the proposed method is about $16.2 \%$ and $2.4 \%$ lower than that of the static model and adaptive model with a fixed moving window, respectively.

For the training time, it can be seen that the average training time of comparative methods is 0.17 seconds, 1.92 seconds, and 0.32 seconds, respectively. The average testing time of comparative methods is 0.10 milliseconds, 0.29 milliseconds, and 0.27 milliseconds, respectively. The training and testing time of static weighted OCSVM is less than that of adaptive models. The reason is that the number of training samples of adaptive models will increase with each additional chunk of data.

Comparing the AR, FAR, training time, and testing time of the three weighted OCSVM-based fault detection model, it can be seen that the AR and FAR of the proposed method achieve the best performance among all the methods. The training time and testing time of the static model achieve the best performance among all the methods. But when the number of testing samples is 200, the average testing time of the proposed method is about 0.27 milliseconds. It can be seen that the adaptive weighted OCSVM can detect testing samples with high calculated speed.

4.3. Detection Performance of Adaptive Weighted SVDD. Similar to the initial setting in the previous section, the turbine engine disk also runs normally at 5000 RPM for 9000 samples and then switched to a notch model for 9000 samples (3000 samples with a rotating speed of 5000 RPM, 4000 RPM, and 3000 RPM, respectively). Several kinds of fault detection methods, that is, static weighted one-class SVDD, adaptive weighted one-class SVDD with fixed moving window, and adaptive weighted one-class SVDD with incremental and decremental strategy, are compared. The settings of the three weighted one-class SVDD are similar to the previous section. The average and standard deviation of AR and FAR, the average training time, and the average testing time of the comparative algorithms are recorded in Table 2. Additionally, the detailed AR and FAR behaviors of the different fault detection models on each data chunk are shown in Figure 5.

From Table 2 and Figure 5, it is observed that the detection performance of adaptive weighted SVDD with incremental and decremental strategy is compared favorably to the other algorithms. In general, it achieves the best performance of all methods.

From Figure 5(a), we can see that the detection accuracy rate is almost $100 \%$ after 9000 testing samples. After 9000 testing samples, there is a notch in the turbine engine disk, which operates in abnormal working mode. The weight SVDD-based fault detection model is an unsupervised learning method for constructing a separating hyperplane which means that only normal samples are used to build the detection model. Therefore, by training enough normal samples for weighted SVDD, the detection rate of abnormal samples can be effectively improved.

From Table 2, it can be clearly seen that the average AR of three weighted OCSVM-based fault detection methods is $87.2 \%, 98.2 \%$, and $99.2 \%$, respectively. The average AR of the proposed method is about $12 \%$ and $1.0 \%$ higher than that of the static model and adaptive model with a fixed moving window, respectively. The FAR of three weighted OCSVMbased fault detection methods is $18.0 \%, 3.7 \%$, and $1.6 \%$, respectively. The average FAR of the proposed method is 
TABLE 1: Comparison of different weighted OCSVM methods.

\begin{tabular}{lcccc}
\hline Detection model & AR (\%) & FAR (\%) & Training time (s) & Testing time (ms) \\
\hline Static weighted OCSVM & $91.0 \pm 9.7$ & $18.0 \pm 4.8$ & $\mathbf{0 . 1 7}$ & $\mathbf{0 . 1 0}$ \\
Adaptive weighted OCSVM with fixed moving window & $98.0 \pm 2.8$ & $4.2 \pm 2.7$ & 1.92 & 0.29 \\
Adaptive weighted OCSVM with incremental and decremental strategy & $\mathbf{9 9 . 1} \pm \mathbf{3 . 0}$ & $\mathbf{1 . 8} \pm \mathbf{4 . 0}$ & 0.32 & 0.27 \\
\hline
\end{tabular}

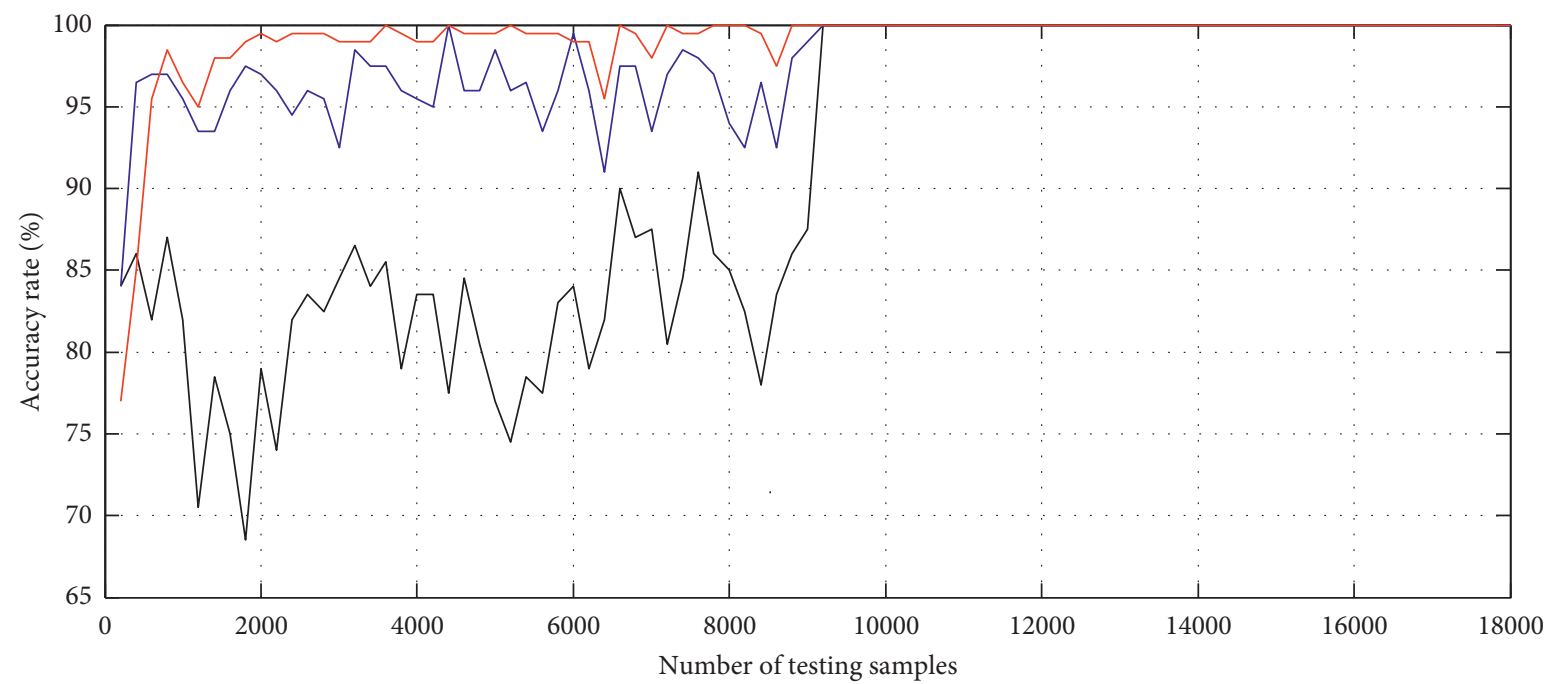

— Static weighted OCSVM

_ Adaptive weighted OCSVM with fixed moving window

_ Adaptive weighted OCSVM with incremental and decremental strategy

(a)

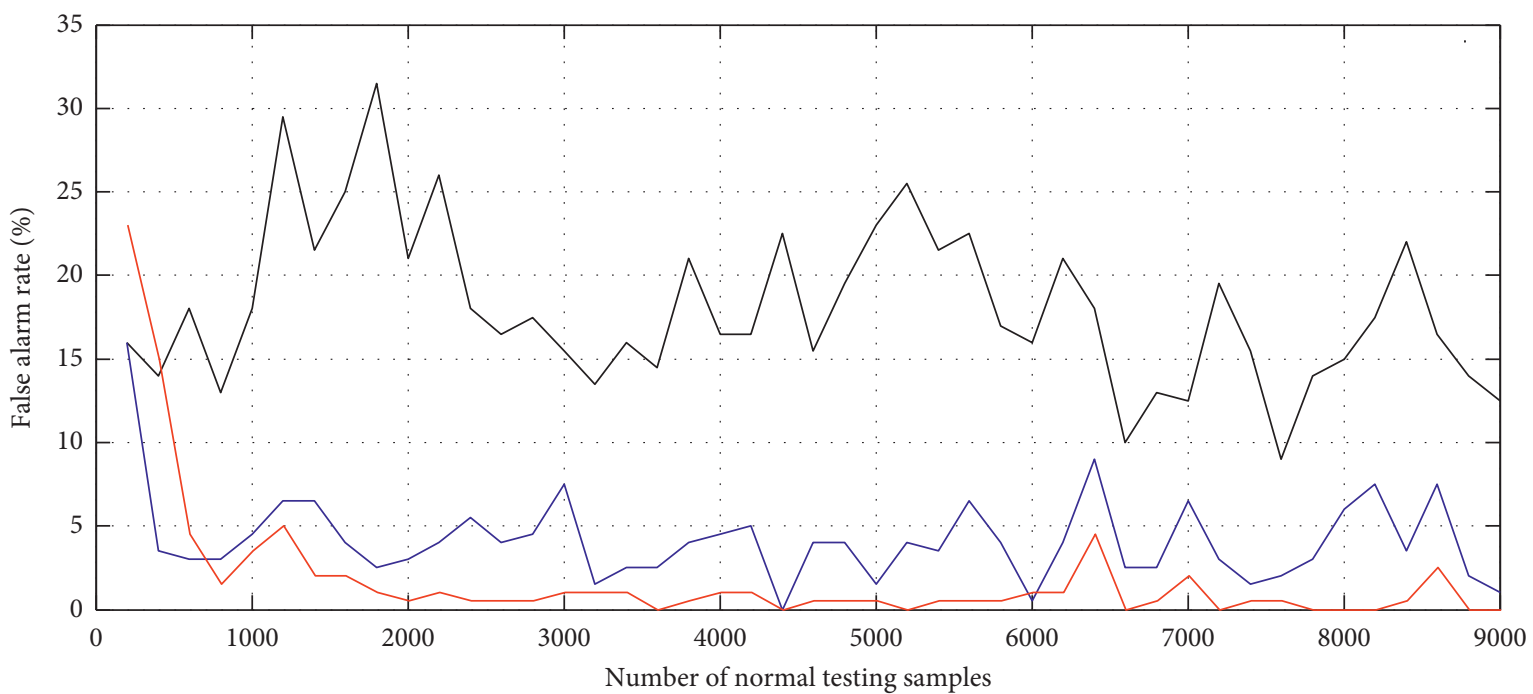

- Static weighted OCSVM

_ Adaptive weighted OCSVM with fixed moving window

_ Adaptive weighted OCSVM with incremental and decremental strategy

(b)

FIgURE 4: AR and FAR on three weighted OCSVM methods. (a) Accuracy rate. (b) False alarm rate.

about $16.4 \%$ and $2.1 \%$ lower than that of the static model and adaptive model with a fixed moving window, respectively.
For the training time, it can be seen that the average training time of comparative methods is 0.13 seconds, 1.38 seconds, and 0.26 seconds, respectively. The average testing 
TABLE 2: Comparison of different weighted SVDD methods.

\begin{tabular}{lcccc}
\hline Detection model & AR (\%) & FAR (\%) & Training time (s) & Testing time (ms) \\
\hline Static weighted SVDD & $87.2 \pm 10.0$ & $18.0 \pm 5.1$ & $\mathbf{0 . 1 3}$ & $\mathbf{0 . 1 0}$ \\
Adaptive weighted SVDD with a fixed moving window & $98.2 \pm 2.7$ & $3.7 \pm 2.8$ & 1.38 & 0.27 \\
Adaptive weighted SVDD with incremental and decremental strategy & $\mathbf{9 9 . 2} \pm \mathbf{2 . 8}$ & $\mathbf{1 . 6} \pm \mathbf{3 . 8}$ & 0.26 & 0.31 \\
\hline
\end{tabular}

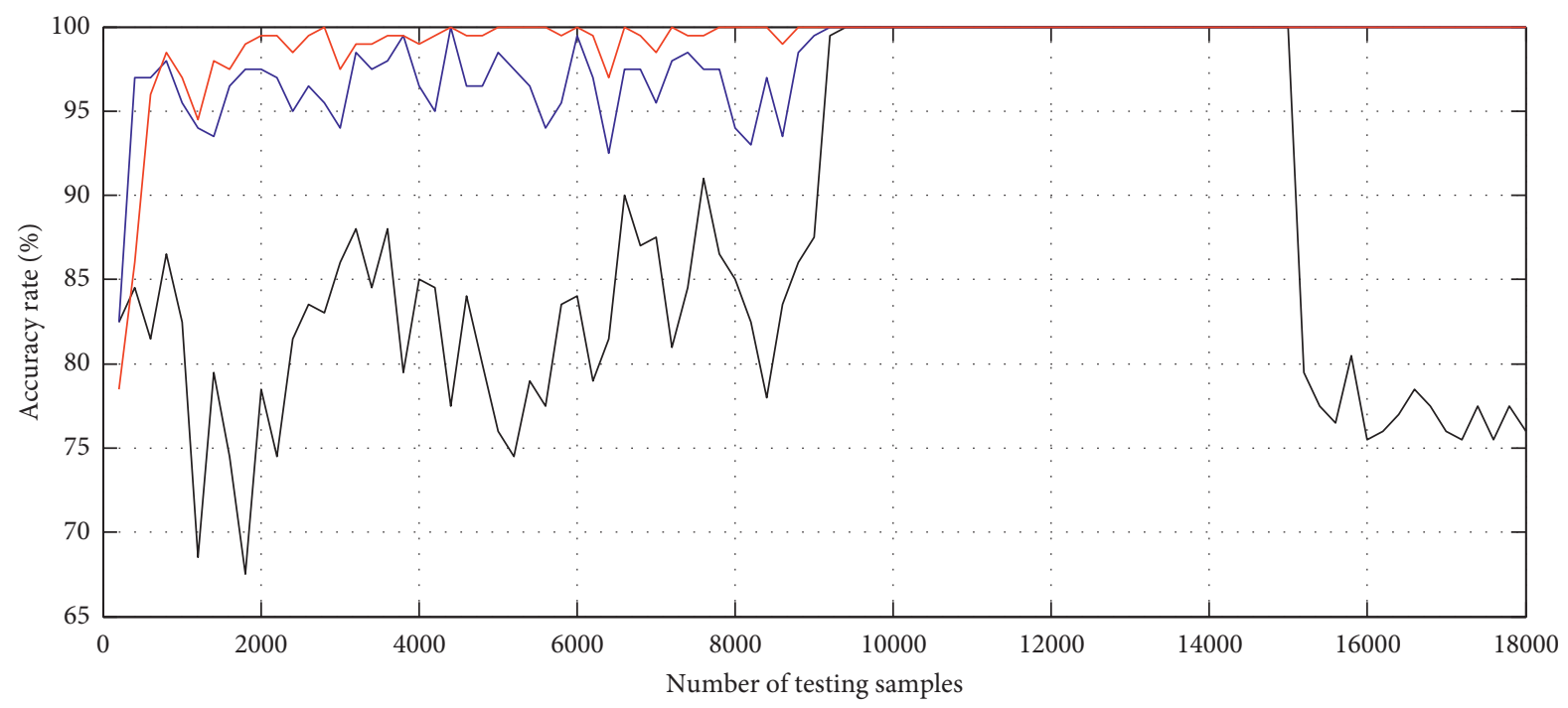

— Static weighted SVDD

_ Adaptive weighted SVDD with fixed moving window

_ Adaptive weighted SVDD with incremental and decremental strategy

(a)

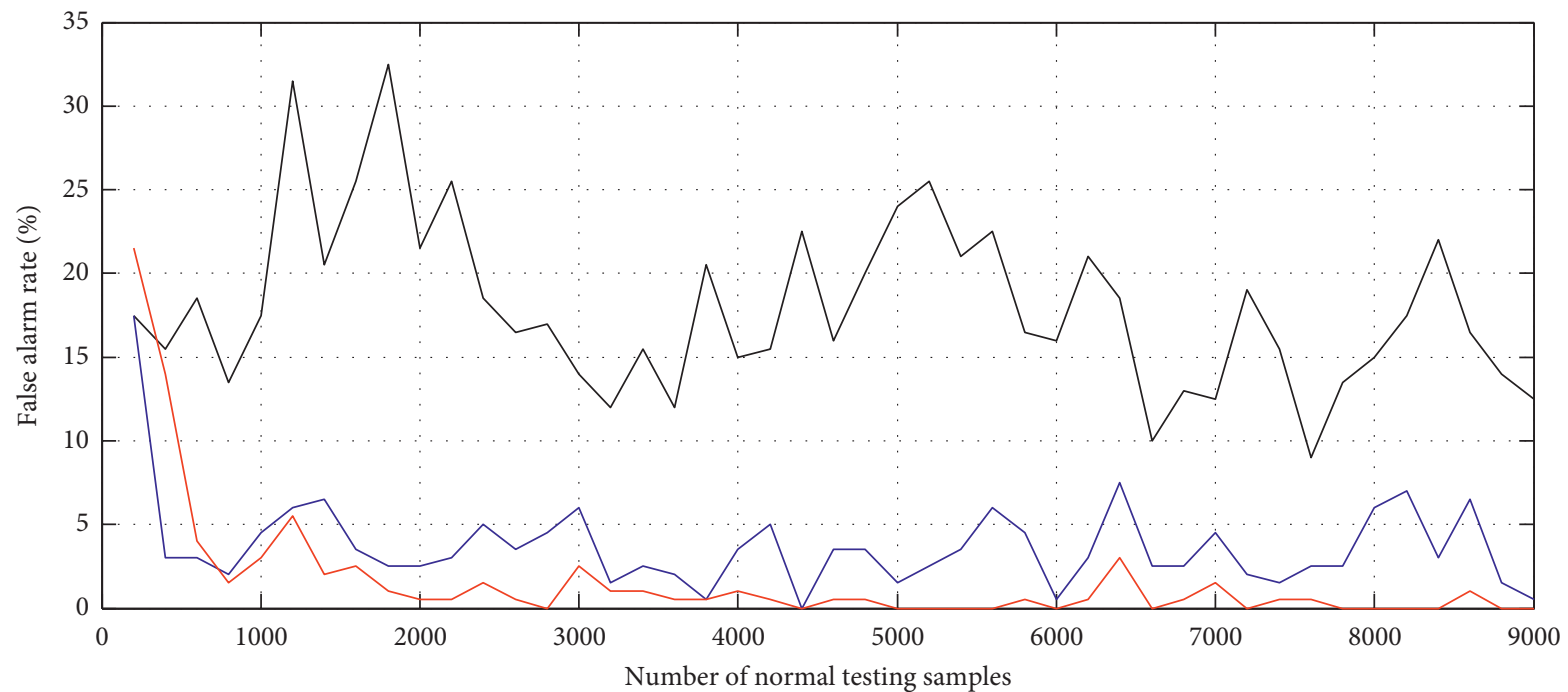

Static weighted SVDD
Adaptive weighted SVDD with fixed moving window
Adaptive weighted SVDD with incremental and decremental strategy

(b)

FIgURE 5: AR and FAR on three weighted SVDD methods. (a) Accuracy rate. (b) False alarm rate.

time of comparative methods is 0.10 milliseconds, 0.27 milliseconds, and 0.31 milliseconds, respectively. The training and testing time of static weighted SVDD is less than that of adaptive models. The reason is that the number of training samples of adaptive models will increase with each additional chunk of data. 
Comparing the AR, FAR, training, and testing time of three weighted SVDD-based fault detection model, it can be seen that the AR and FAR of the proposed method achieve the best performance among all the methods. The training time and testing time of the static model achieve the best performance among all the methods. But when the number of testing samples is 200, the average testing time of the proposed method is about 0.31 milliseconds. It is evident that the adaptive weighted one-class SVDD can detect testing samples with high calculated speed.

By comparing all the listed detection performances of the test cases, the following conclusion could be drawn:

(i) The detection performance of the adaptive weighted one-class SVM detection model with incremental and decremental strategy is significantly better than that of the static model and adaptive model with the fixed moving window. In the proposed incremental learning, the new incoming data represents the current state of the turbine engine disk and the support vectors represent the historical state of the classifier mix together to increase the detection performance. Additionally, the new weight of the training sample is calculated based on the distance between the sample and the decision boundary. The highest weight for incoming samples represents the alternative in the decision boundary which shows a significant impact on the new decision boundary. This is attributed to the fact that the adaptive weighted one-class SVM based on the proposed incremental strategy can obtain a more accurate classifier

(ii) With the decremental strategy based on KNN, the proposed decremental strategy can speed up the adaptive fault detection model while still guaranteeing correct results. The basic structure of input samples is preserved by computing the nearest neighbors and the redundant data is reduced by preserving the pair of samples with the maximum Euclidean distance. Therefore, the decremental strategy based on KNN does not change the basic structure of input samples, which could efficiently improve the robustness of the algorithm.

\section{Conclusion}

In this paper, an adaptive weighted one-class SVM algorithm with incremental and decremental strategy is proposed for fault detection of the turbine engine disk.

The proposed method can adaptively modify the weight of the training samples by incremental training, in which significant weight samples represent the changeable of the decision boundary. Additionally, with the decremental strategy based on KNN, the proposed decremental strategy can accelerate the calculated speed of the adaptive weighted one-class SVM detection model. Comprehensive experiments and comparisons have validated the effectiveness of the proposed approach. But the training and testing time of the proposed method is more than that of the static model.
The reason is that the number of training samples of adaptive models will increase with each additional chunk of data. In the future, it will be interesting to use the idea of adaptive weighted SVM to solve the drifting problem in a time series data stream with imbalanced data.

\section{Data Availability}

The Disk Defect Data are available at https://c3.nasa.gov/ dashlink/resources/314/. The data presented are from a physical simulator that simulated engine disks. All parameters are sampled once per revolution measuring 32 blades to edge clearances on each revolution. Data are recorded for approximately 3 minutes for each run. The parameters are the revolution measurement; the clock time; the measured RPM; the maximum, average, and minimum gap across all gap sensors; and the measured gap for blade 0 to blade 31 . Faults include medium and large cracks on the disk.

\section{Conflicts of Interest}

The authors declare that they have no conflicts of interest.

\section{Acknowledgments}

This work was supported by the National Natural Science Foundation of China under grant nos. 61603395 and U1833108.

\section{References}

[1] P. Yu, D. Zhang, Y. Ma, and J. Hong, "Dynamic modeling and vibration characteristics analysis of the aero-engine dual-rotor system with Fan blade out," Mechanical Systems and Signal Processing, vol. 106, pp. 158-175, 2018.

[2] B. Zhao, L. Xie, H. Li, S. Zhang, B. Wang, and C. Li, "Reliability analysis of aero-engine compressor rotor system considering cruise characteristics," IEEE Transactions on Reliability, vol. 69, no. 1, pp. 245-259, 2020.

[3] C. Karlsson, J. Arriagada, and M. Genrup, "Detection and interactive isolation of faults in steam turbines to support maintenance decisions," Simulation Modelling Practice and Theory, vol. 16, no. 10, pp. 1689-1703, 2008.

[4] L. Sivakumar and S. Devi, "Implementation of VLSI model as a tool in diagnostics of slowly varying process parameters which affect the performance of steam turbine," Applied Soft Computing, vol. 24, pp. 730-741, 2014.

[5] A. Abdul-Aziz, M. R. Woike, N. C. Oza, B. L. Matthews, and J. D. lekki, "Rotor health monitoring combining spin tests and data-driven anomaly detection methods," Structural Health Monitoring: An International Journal, vol. 11, no. 1, pp. 3-12, 2011.

[6] A. Abdul-Aziz, M. R. Woike, R. C. Anderson, and K. Aboumerhis, "Propulsion health monitoring assessed by microwave sensor performance and blade tip timing," in Proceedings of the Smart Structures and NDE for Energy Systems and Industry 4.0, Denver, CO, USA, March 2019.

[7] N. Saravanan and K. I. Ramachandran, "Incipient gear box fault diagnosis using discrete wavelet transform (DWT) for feature extraction and classification using artificial neural network (ANN)," Expert Systems With Applications, vol. 37, no. 6, pp. 4168-4181, 2010. 
[8] I. Ferreira Santos and I. F. Santos, "Comparative analysis of neural network and regression based condition monitoring approaches for wind turbine fault detection," Mechanical Systems and Signal Processing, vol. 25, no. 5, pp. 1849-1875, 2011.

[9] P. Ravi Kumar, D. Infield, and A. Kolios, "Comparison of advanced non-parametric models for wind turbine power curves," IET Renewable Power Generation, vol. 13, no. 8, pp. 1503-1510, 2019.

[10] R. K. Pandit and D. Infield, "Comparative analysis of binning and gaussian process based blade pitch angle curve of a wind turbine for the purpose of condition monitoring," Journal of Physics: Conference Series, vol. 1102, Article ID 012037, 2018.

[11] C. Li, R.-V. Sanchez, G. Zurita et al., "Gearbox fault diagnosis based on deep random forest fusion of acoustic and vibratory signals," Mechanical Systems and Signal Processing, vol. 76-77, pp. 283-293, 2016.

[12] V. Cherkassky and Y. Ma, "Practical selection of SVM parameters and noise estimation for SVM regression," Neural Networks, vol. 17, no. 1, pp. 113-126, 2004.

[13] L. B. Jack and A. K. Nandi, "Fault detection using support vector machines and artificial neural networks, augmented by genetic algorithms," Mechanical Systems and Signal Processing, vol. 16, no. 2-3, pp. 373-390, 2002.

[14] R. K. Pandit and D. Infield, "Comparative assessments of binned and support vector regression-based blade pitch curve of a wind turbine for the purpose of condition monitoring," International Journal of Energy and Environmental Engineering, vol. 10, no. 2, pp. 181-188, 2019.

[15] N. Laouti, N. Sheibat-Othman, and S. Othman, "Support vector machines for fault detection in wind turbines," IFAC Proceedings Volumes, vol. 44, no. 1, pp. 7067-7072, 2011.

[16] A. Anaissi, N. L. D. Khoa, T. Rakotoarivelo, M. M. Alamdari, and Y. Wang, "Adaptive online one-class support vector machines with applications in structural health monitoring," ACM Transactions on Intelligent Systems and Technology, vol. 9, no. 6, pp. 1-20, 2018.

[17] S. Lee and S. B. Kim, "Time-adaptive support vector data description for nonstationary process monitoring," Engineering Applications of Artificial Intelligence, vol. 68, pp. 1831, 2018.

[18] B. Krawczyk and M. Woźniak, "One-class classifiers with incremental learning and forgetting for data streams with concept drift," Soft Computing, vol. 19, no. 12, pp. 3387-3400, 2014.

[19] B. Krawczyk and M. Woźniak, "Incremental weighted oneclass classifier for mining stationary data streams," Journal of Computational Science, vol. 9, pp. 19-25, 2015.

[20] M. Bicego and M. A. T. Figueiredo, "Soft clustering using weighted one-class support vector machines," Pattern Recognition, vol. 42, no. 1, pp. 27-32, 2009.

[21] B. Schölkopf, R. Williamson, A. Smola, J. Shawe-Taylor, and J. Platt, "Support vector method for novelty detection," in Advances in Neural Information Processing Systems-NIPS 1999, pp. 582-588, MIT Press, Cambridge, MA, USA, 1999.

[22] D. M. J. Tax and R. P. W. Duin, "Support vector data description," Machine Learning, vol. 54, no. 1, pp. 45-66, 2004.

[23] J. Chen, X. Zhang, and Y. Gao, "Fault detection for turbine engine disk based on an adaptive kernel principal component analysis algorithm," Proceedings of the Institution of $\mathrm{Me}$ chanical Engineers, Part I: Journal of Systems and Control Engineering, vol. 230, no. 7, pp. 651-660, 2016.

[24] C.-C. Chang and C.-J. Lin, "Libsvm," ACM Transactions on Intelligent Systems and Technology, vol. 2, no. 3, pp. 1-27, 2011.
[25] M.-W. Chang, H.-T. Lin, M.-H. Tsai, C.-H. Ho, and H.-Fu Yu, "Weights for data instances," July 2019, https://www.csie.ntu. edu.tw/ cjlin/libsvmtools/\#weights_for_data_instances. 